\title{
Generalizing a Quantum-well Infrared Single-pixel Polarimeter (QWISPP) to Fourier Transform Spectral-Polarimetric Imaging
}

\author{
Mario Serna and Dustin McCauley \\ US Air Force Academy, Department of Physics \\ 2354 Fairchild Drive, Suite 2A153 USAF Academy, CO 80840-6254, USA
}

\begin{abstract}
At previous SPIE meetings, we reported on an optoelectronic device that measures the complete polarization state of incident infrared light in a single pixel and in a single frame for a narrow wavelength band $(\delta \lambda<0.05$ $\mu \mathrm{m})$. Using at least four quantum-well stacks and four linear gratings, each stacked alternating above the other, the device uses the interference among light paths to create a distinct pattern of photocurrents at each quantum-well stack coding for a specific polarization. In this paper, we will model the performance of this device, a quantum-well infrared single-pixel polarimeter (QWISPP), in the setting of a Fourier transform infrared (FTIR) imager. We model one column of QWISPP pixels detecting an interferogram. Using an FTIR with randomly varying QWISPP pixels to detect the interferogram, we discovered a technique that allows an $100 \times$ improvement in measured spectral-polarization uncertainty compared to the use of identical QWISPP pixels in an FTIR or grating spectrometer. The technique also enables a $15 \times$ improvement in the uniformity of the error across a sample spectrum. In other words, we turn into an advantage the imperfections in fabricating an FPA of QWISPPs.
\end{abstract}

Keywords: FTIR imager, polarimeter, coregistered, Stokes vectors, remote sensing, spectral polarization detector, polarization uncertainty

\section{INTRODUCTION}

With spectro-polarimetric imaging, one can remotely sense more information than with just spectral imaging. There are a multitude of potential military and civilian advantages to spectropolarimetric sensors that can be found in the cited references. ${ }^{1-4}$ In this paper, I will focus on a new type of spectro-polarimetric imaging that combines the QWISPP pixel described in references ${ }^{5-7}$ with the FTIR imaging like that used in references. ${ }^{8,9}$

The next few paragraphs should answer the question: "What is special about a QWISPP?" Polarization is normally detected by combining a polarization filter, like polarized sunglasses, with a spectrally sensitive detector, like a digital camera or the human eye. With these tools, one can only detect one polarization component at a time for the spectral range of the camera. To learn the actual polarization state, one needs to compare multiple images taken with different orientations or rotations of the filter to extract the four parameters needed to define the polarization state. A common basis to represent the polarization state is the Stokes vector: $S_{0}=I_{0}+I_{90}, S_{1}=I_{0}-I_{90}, S_{2}=I_{45}-I_{135}$, and $S_{3}=I_{R}-I_{L}$ where $I_{X}$ is the measured intensity of the light after passing through a linear filter at an orientation of $X$ degrees, and $I_{R}$ and $I_{L}$ are the measured intensities of right or left circularly polarized fraction of the light. A normalized Stokes parameter is simply the Stokes parameter $S_{j}$ divided by $S_{0}$.

Naively, one may think no more than two of the four parameters that describe the polarization can be extracted serially from a beam of light. One parameter can be found by measuring the absorption by

Send correspondence to Mario Serna

E-mail: mariojr@alum.mit.edu, Telephone: 17193339733 
one filter, and a second parameter by measuring the absorption of a complimentary filter directly after the first filter. To challenge this misconception, we introduced in previous publications the quantum-well infrared single-pixel polarimeter (QWISPP) that will detect all four components of the polarization at each wavelength in a single pixel and in a single frame. ${ }^{5-7}$

In these previous papers, we reviewed other polarimeters that detect the polarization over spatially separated pixels or by comparing images taken over time, ${ }^{3,10,11}$ and we reviewed the importance of pixel registration for accurate polarization measurements. ${ }^{12}$ Since then, we have learned of a another polarimeter design by Van Delden. ${ }^{13}$ In this new design the light from an individual pixel and wavelength is spread over an entire focal plane array (FPA), and spatial interference across a 2D focal plane array is used to encode the polarization. Their device operates at a single narrow spectral band. To form an image, one has to scan point by point across the image.

A QWISPP is special and distinct from all previous polarimeters because it is the only device that detects in a single physical pixel the four Stokes parameters. The QWISPP is a counter example to the misconception that only two polarization parameters can be extracted serially from a beam of light; it eliminates registration error; and it provides significant size and weight savings by reducing the number of pixels, beam splitters, and optics by a factor of four.

Despite this unique set of capabilities, a QWISPP is not without problems. In addition to being extremely difficult to build, ${ }^{7}$ the physics of the QWISPP design makes the performance sensitive to more than 17 parameters, including the wavelength of the incident light, the physical size of the QWISPP grating periods, the separation between gratings, and other structural dimensions. The QWISPP operates on interference between light paths that diffract in all possible ways between the four sets of gratings. Because the interference depends on the wavelength, the device must be illuminated by a narrow wavelength band $(\delta \lambda<0.05 \mu \mathrm{m})$ to give a clean response that can be used to recover the polarization.

A single QWISPP pixel will have a widely varying performance at different wavelengths. The left side of figure 1 shows the recovered uncertainty in normalized Stokes parameters for a single QWISPP structure tiled across the FPA of a grating spectrometer. The QWISPP detects each Stokes parameters with a different uncertainty at each wavelength. At some wavelengths, uncertainty is as low as 0.5 percent of a normalized Stokes parameter. At other wavelengths, the uncertainty is 200 percent, twice as big as the signal.

In our previous publications, we suggested these characteristics meant the QWISPP needed to be used in combination with a push-broom grating spectrometer. In a grating spectrometer, the unfortunate manufacturer would have the formidable task of optimizing each pixel for the wavelength incident at that position on the FPA. The reality of semiconductor fabrication makes it practically impossible to fabricate an optimized pixel in the correct position of an FPA. In this paper, we will show how to use an FTIR configuration and the random pixel-to-pixel variation of the FPA to improve the average spectro-polarimetric uncertainty by $100 \times$ and the uniformity in that uncertainty by $15 \times$. The right and side of figure 1 demonstrates this improvement. In other words, we turn into an advantage the imperfections in fabricating an FPA of QWISPPs.

The reader may ask: if the original purpose of the device was to detect all the information on a single pixel, then why do we want to use an FTIR configuration where the information is spread over a linear column of pixels? Even though we are distributing the detection over a column of QWISPP pixels, the QWISPP pixels still eliminate registration errors and enable at least a $4 \times$ improvement in spectral-polarization resolution for a given optics size and weight and a given FPA size when compared to the designs in references. ${ }^{3,10,11,13}$ The use of QWISPP pixels also enables circular polarization detection which is problematic with other snapshot polarimeter designs. ${ }^{10}$

\section{THE QWISPP PIXEL}

The QWISPP shown in figure 2 has four readouts from quantum-well stacks layered one above the other and separated by linear gratings at various orientations; at least four layers are required for full polarimetric detection. The gratings can be metal-semiconductor ${ }^{5}$ or dielectric-semiconductor. ${ }^{7,14}$ The pixel- 

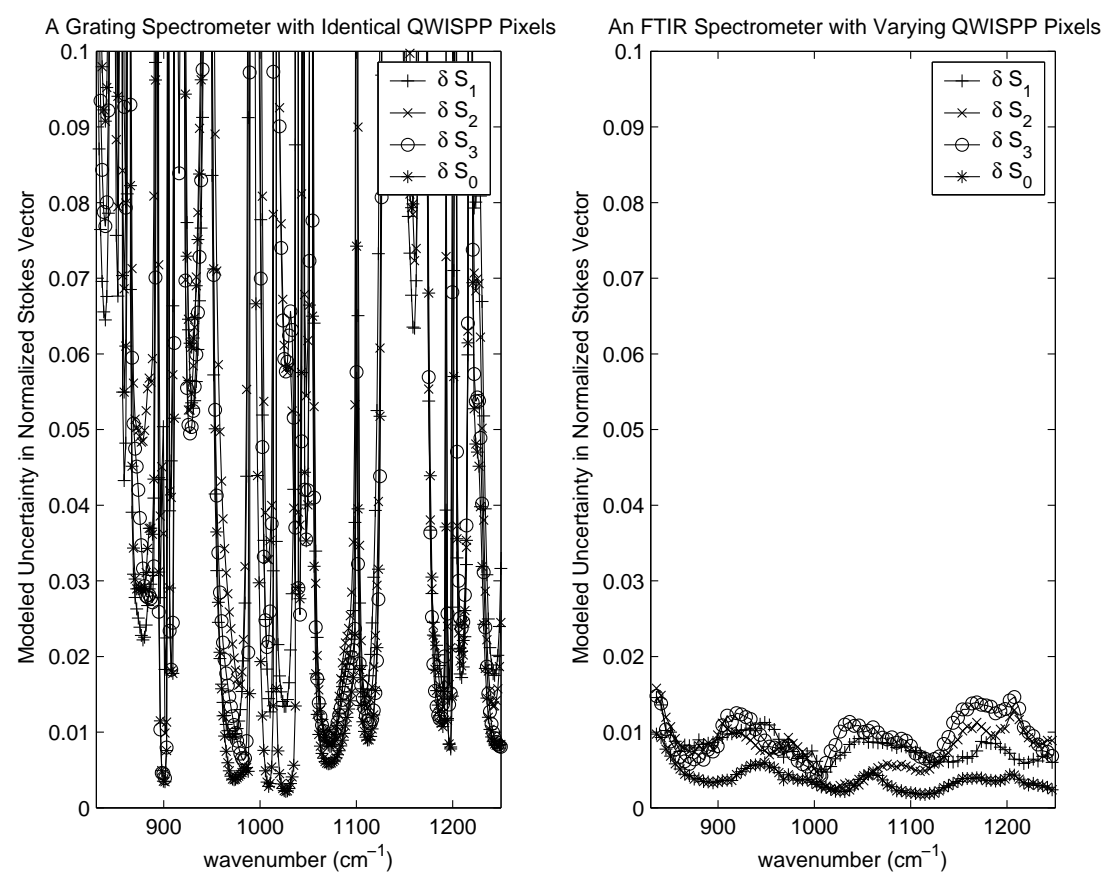

Figure 1. A side-by-side comparison of the error in determining the normalized Stokes parameters for a grating spectrometer using an FPA with all identical QWISPP pixels versus an FTIR spectrometer using an FPA with randomly varying QWISPP pixels. The left figure shows how a single QWISPP structure will be able to measure the Stokes parameters with varying uncertainty at different wave numbers. The right figure shows the performance improvement from an FTIR configuration with random pixel variations.
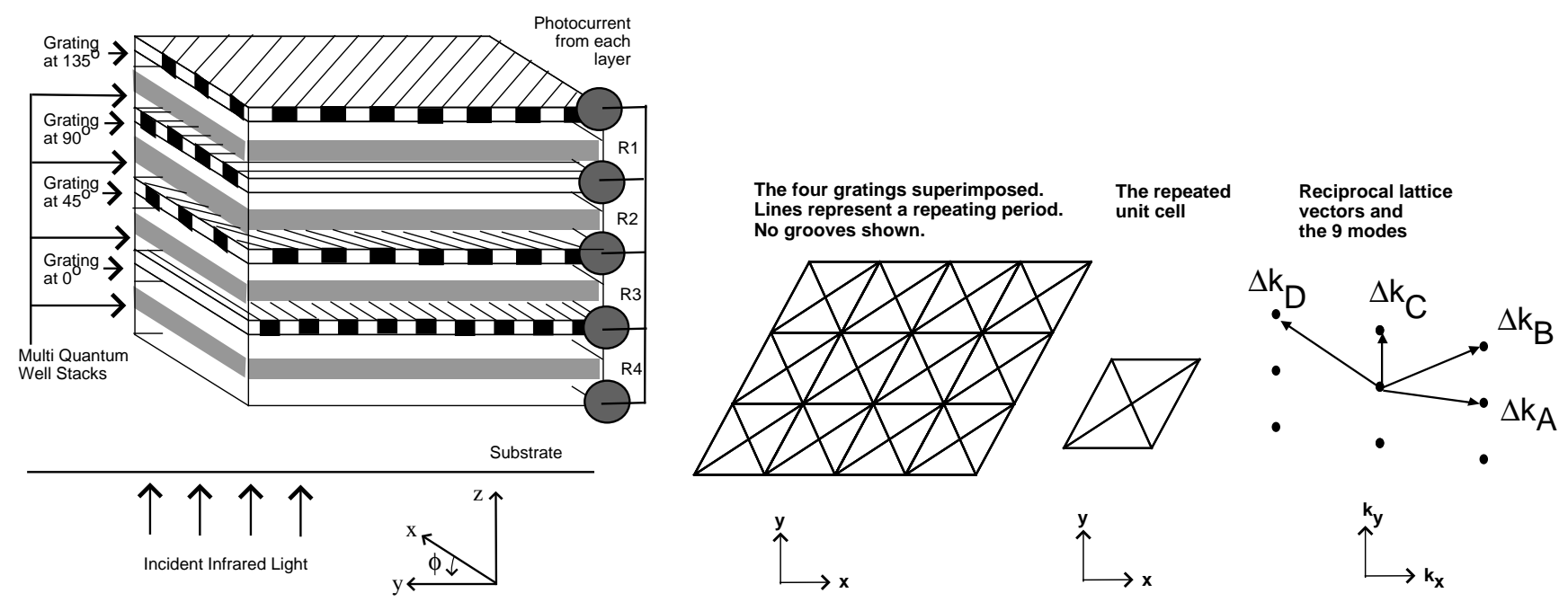

Figure 2. Each quantum-well infrared single-pixel polarimeter (QWISPP) consists of four (or more) quantum-well stacks that can be individually read and each separated by linear gratings at different orientations. To facilitate the modeling of the device, the periods of the four layers are chosen such that they form a repeating unit cell. 
polarimeter device detects the incident light's polarization by exploiting the polarization-dependent absorption of quantum-well infrared photodetectors, the polarization-dependent diffraction from linear gratings, and the polarization-dependent interference of electromagnetic waves.

Incident light $S^{i n}$ on a QWISPP pixel will produce a pattern of photocurrents $R$. The equation

$$
R_{j}=\sum_{k=1}^{N_{R}} M_{j i}(k) S_{i}^{\mathrm{in}}(k)
$$

shows this pattern of photocurrents $R_{j}$ is a linear map from the Stokes vector $S_{i}^{\text {in }}(k)$ with a wave vector $k=2 \pi / \lambda$, or equivalently a wavelength $\lambda$, to the readouts (also called photocurrents) given by the vector $R_{j}$ where the indices $j$ and $i$ run through the dimensions of the respective vectors. These two vectors are related by a matrix $M$ that maps the four components of the incident light's Stokes vector $S_{i}^{\text {in }}$ to the four (or more) photocurrents $R_{j}$ where the index $j$ runs from 1 to the number of readouts $N_{R}$. For all the calculations in this paper, $N_{R}=4$. One can further decrease the error compared to the results of this paper by letting $N_{R}>4$ and designing the structure with a grating material that allows more light to penetrate to the deepest layers of the device. ${ }^{14}$ The matrix $M$ will be called the device's polarization response matrix, and in our model, $M$ depends on more than 17 parameters including the structure of the pixel, the wavelength of the incident light, and the incidence angle of the incident light. The dependence on all these parameters is suppressed for simplicity.

The components of $M$ at a spectral line can be found by calibrating the pixel polarimeter's response to polarized and unpolarized radiation at the spectral frequency. In this paper, we use a numerical model and not an actual device. For example: subtracting the readouts $R_{j}^{I}$ that result from unpolarized light from the readouts $R_{j}^{V}$ of vertically polarized light gives the components of the $2^{\text {nd }}$ column of the $M$ matrix: $M_{j 2}=R_{j}^{I}-R_{j}^{V}=V_{j}^{2}$ where $V^{2}$ is the Stokes basis vector for $S_{2}$ in the readout vector space. In this way, the matrix $M$ can be determined at each wavelength by means of measuring the device's response to incident light.

By propagating uncorrelated errors from the different readouts, the device's uncertainty in recovering the Stokes vectors $\delta S_{j}$ given an uncertainty in the $k^{\text {th }}$ readouts $\delta R_{k}$ is given by

$$
\delta S_{j}=\sqrt{\sum_{k=1}^{N_{R}}\left|\left(M^{-1}\right)_{j k}\right|^{2}\left(\delta R_{k}\right)^{2}} .
$$

If the uncertainty in the different readouts is equal, this expression can be simplified to

$$
\delta S_{j}=\Lambda_{j} \delta R \quad \text { where } \quad \Lambda_{j}=\sqrt{\sum_{k=1}^{N_{R}}\left|\left(M^{-1}\right)_{j k}\right|^{2}}
$$

$\Lambda_{j}$ is the device's inherent polarization uncertainty, and $\delta R$ is the common uncertainty of each readout. In the previous section, figure 1 shows $\delta S_{j}$ at spectrum of wave numbers for a single QWISPP structure.

The QWISPP models used for all the results, including figure 1 in the previous section, share common parameters except where noted. To find $\delta S_{j}$, we always use $\delta R=0.000116$ for reasons explained in section 3.1. All use perfectly conducting square gratings with a depth of 0.3 microns and a dielectric width of 0.7 times the period. The second grating that incident light will encounter always has a period of 3.13 microns. The top reflective grating has a period of 2.89 microns for the results on the left side of figure 1 . On the right side of figure 1 and in section 3.4, the top reflective period is randomly varied following a normal distribution with a mean of $3.13 \mu \mathrm{m}$ and a standard deviation of $0.2 \mu \mathrm{m}$. The remaining two periods and angles are fixed by the necessity to form a repeating unit cell. As shown in figure 2, the need for a repeating unit cell constrains the number of modes to a finite, easy to model, set. The modeled quantum wells were formed 
with GaAs / AlGaAs, and follow the algorithm given by Andersson and Lundqvist. ${ }^{15,16}$ The separation between successive gratings is 2.75 microns. The model was written in GNU $\mathrm{C}++$ and scripts to run the code repeatedly were written in MatLab. The source code or the executable is available upon request via e-mail.

\section{MODELING AN IMAGING FTIR COLUMN OF PIXELS}

In section 3, we will first quantify the readout error, and we will define the assumptions and methodology for modeling the QWISPP FTIR. Next, we will discuss the process of recovering the spectral-polarization and associated uncertainty for the case of identical QWISPP pixels in an imaging FTIR and finally for the case of QWISPP pixels with random structural variations in an imaging FTIR.

\subsection{Readout error due to the electronics}

The source of all uncertainties shown in this paper is readout error or equivalently error in reading the photocurrent. We neglect a small error due to the modeling assumption that the polarization response matrix is identical for the two impinging beams. Later in this subsection, we will show this error source is negligible for interferograms formed from beams with an angle separation smaller than 0.03 degrees. In short, we will group all error in the readouts as $\delta R \approx 0.000116$ for impinging light of unit intensity. The remainder of subsection 3.1 justifies these statements.

Using current quantum-well infrared photodetector (QWIP) cameras as a reference, ${ }^{17,18}$ an FPA can be fabricated with a noise-equivalent temperature difference $N E \Delta T=9 \mathrm{mK}$ for signal at room temperature $T=300$ Kelvin near a wavelength of $\lambda_{o} \approx 10 \mu \mathrm{m}$. Assuming the photocurrent response is linear in the intensity of the signal, we can find an expression for the signal to noise ratio $R / \delta R^{e}$ from the definition of $N E \Delta T$, which is the largest temperature difference that cannot be distinguished from noise. With this definition, the signal-to-noise ration is equivalent to

$$
\frac{I\left(\lambda_{o}, T_{o}\right)}{I\left(\lambda_{o}, T_{o}+N E \Delta T\right)-I\left(\lambda_{o}, T_{o}\right)}=\frac{R}{\delta R^{e}} \approx 6900
$$

where $I\left(\lambda_{o}, T\right)$ is the black body intensity at wavelength $\lambda_{o}$ and temperature $T$. We are measuring the readouts in terms of the amount of the incident light absorbed. This means that in our model and in all our plots in this paper, the readouts have units of intensity. Let's assume the QWIP considered in references ${ }^{17,18}$ absorbed around $80 \%$ of the incident light near resonance making $R \approx .8$ when we scale the incident intensity to $S_{0}=1$. Substituting $R$, we find $\delta R^{e}=0.000116$. We will use this value for $\delta R^{e}$ as an estimate of the detector noise for normalized Stokes parameters common to each of the four readouts on each QWISPP.

Another source of error in the model, which we neglect, is the assumption that the polarization response matrix is identical for the two beams of light that form the interferogram. In an FTIR the light is split, and the beam is sent through two paths that end up rejoining with slightly different wave-propagation directions. The polarization response matrix $M$ is a sensitive function of the incident angle of the light and is probably a function of the relative phase between the two beams. Figure 3 shows the 16 components of $M$ as a function of the angle difference between the two beams of light forming the interferogram. We can model the QWISPP FTIR without greatly expanding the QWISPP computer model if we consider only the case where the angle difference is so small that it is smaller than the readout electronics error $\delta R^{e}$. Figure 3 shows this angle to be about $\theta=0.03$ degrees.

We are not loosing any generality by making this assumption. For larger angle separations, the QWISPP will still have a polarization response matrix that can be inverted to recover the "net" polarization from the two beams. The only complication is that this polarization response matrix cannot be calculated separately from the calibration of the pixel from either of the two impinging beams and will depend on the relative phase between these two beams. Because the result depends on the relative phase, the large angle difference basically changes the polarization response matrix from pixel to pixel across the FPA. Therefore, the more realistic case of larger angle differences, $\theta \approx 1.5$ degrees, will be mathematically identical to the case where 

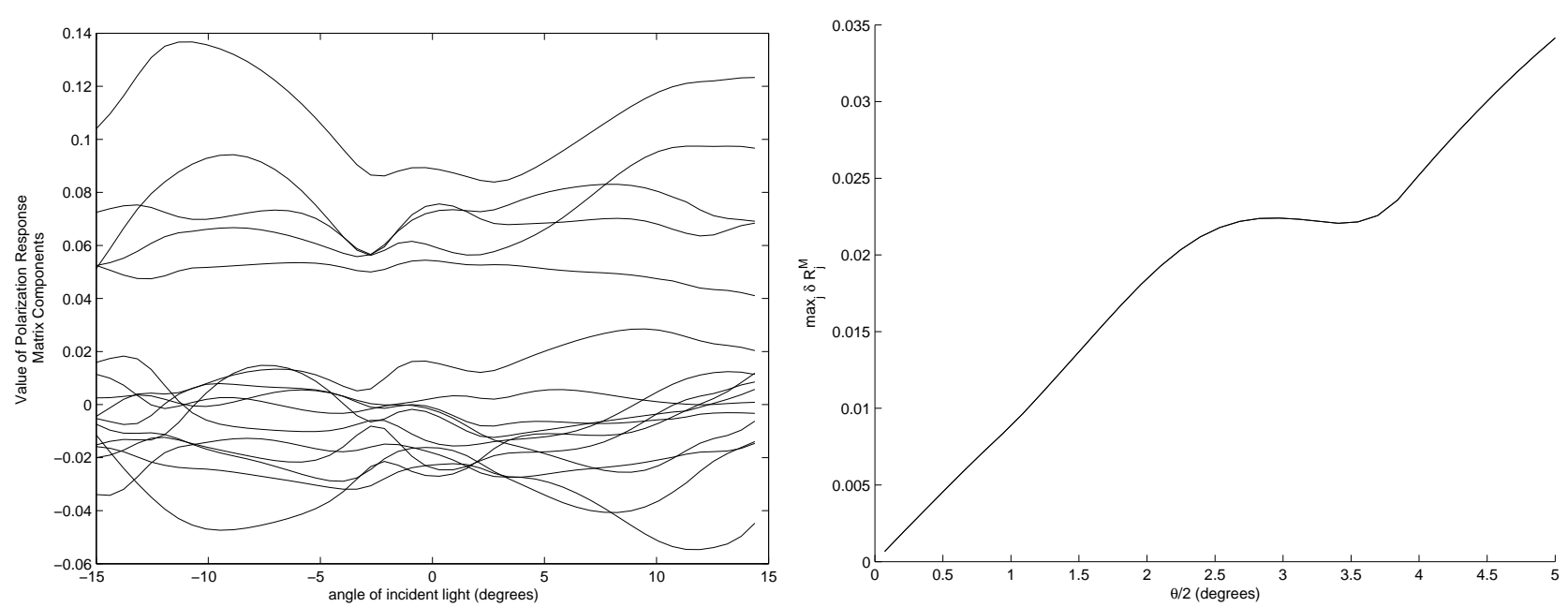

Figure 3. On the left, we show the 16 components of the polarization response matrix $M$ as a function of the angle of incidence for the QWISPP design parameters described in subsection 2. If we assume the two beams that form the interferogram have the same polarization response matrix $M$, then we have a worst case readout uncertainty $\delta R^{M}$ due to the error in the polarization response matrix shown on the right hand side.

the QWISPP pixels on the FPA vary in structure from pixel to pixel. We will study this case where QWISPP structure varies across the FPA in subsection 3.4.

If we assume identical response from two angles of incidence, $-\theta / 2$ and $\theta / 2$, where $\theta$ is the angle difference between the two beams, we will incur an error in the readouts $\delta R^{M}$ due to our choice of the polarization response matrix. The error in the readouts

$$
\left(\delta R_{j}^{M}\right)^{2}=\sum_{k=1}^{4}\left(\delta M_{j k} S_{k}^{i n}\right)^{2}
$$

comes from the error propagation on equation 1 assuming no uncertainty in the incident signal. To simplify the calculations, we assume the two beams, even though they arrive at different angles, give the same polarization response matrix. This introduces an error in $R$ by providing an uncertainty in $M_{j k}$

$$
\left(\delta M_{j k}\right)^{2}=\left(M_{j k}(\theta / 2)-M_{j k}(-\theta / 2)\right)^{2} .
$$

Plugging this uncertainty in $M$ into equation 5 , we fine the total error in the readouts is given by

$$
\left(\delta R_{j}^{T}\right)^{2}=\left(\delta R_{j}^{e}\right)^{2}+\sum_{k=1}^{4}\left(\delta M_{j k} S_{k}^{i n}\right)^{2} .
$$

We used the values from figure 3 to calculate the corresponding worst case uncertainty for $\delta R^{M}$ as a function of half the angle between the two beams $\theta / 2$. The results are also shown in figure 3 . To achieve $\delta R^{M}$ errors smaller than $\delta R^{e}=0.000116$, we will work with $\theta=0.03$ degrees.

Because we have chosen a small angle separation based on the above analysis, we now neglect any contribution from $\delta R^{M}$ to $\delta R$. The conceptual conclusions of the paper do not depend on the exact value of $\delta R$, when calibrating an actual device, one would not have any contribution to $\delta R$ from $\delta R^{M}$. The estimate from $\delta R^{e}$ is sufficient to provide realistic results on which experimental efforts may be based. 


\subsection{Modeling an ideal FTIR}

Our model of the QWISPP FTIR column of pixels makes a few simplifying assumptions. Essentially, we assume an ideal FTIR. The model neglects all imperfections in the optics and the finite size of the pixels. As explained in subsection 3.1, our model also assumes the polarization response matrix is identical for the two incident beams.

We model the interferogram with the simplest possible model. An interferogram forms from light that has been split and recombines at a slight angle. The wave vectors of these two beams are denoted by $\vec{k}_{a}$ and $\vec{k}_{b}$. We set up our axis so that the interferogram is formed along the $y$ axis, and $\vec{k}_{a}$ and $\vec{k}_{b}$ lie in the $z-y$ plane. The light can then be broken up into two modes,

$$
E_{x}(\vec{r})=E_{x}^{a} \exp \left(i \vec{k}_{a} \cdot \vec{r}\right)+E_{x}^{b} \exp \left(i \vec{k}_{b} \cdot \vec{r}\right) \quad \text { and } \quad B_{x}(\vec{r})=B_{x}^{a} \exp \left(i \vec{k}_{a} \cdot \vec{r}\right)+B_{x}^{b} \exp \left(i \vec{k}_{b} \cdot \vec{r}\right)
$$

where $\vec{r}=(x, y, z)$ is the position vector and $E_{x}^{a}, E_{x}^{b}, B_{x}^{a}$, and $B_{x}^{b}$ are complex magnitudes of the $E$ and $B$ modes for the $a$ and $b$ beams which combine to form the interferogram. The two beams have an angle difference of $\theta$ and the focal plane is at $z=0$. The two wave vectors are $\vec{k}_{a}=(0, k \sin (\theta / 2), k \cos (\theta / 2))$ and $\vec{k}_{b}=(0,-k \sin (\theta / 2), k \cos (\theta / 2))$ where $k=\omega / c$. Because both beams originated from a single beam, in an ideal FTIR the coefficients will be related by $E_{x}^{a}=E_{x}^{b}$ and $B_{x}^{a}=B_{x}^{b}$. We will assume the optics do not alter the polarization; this error source could be calibrated out in a real device. A fixed phase shift between the two paths will shift the center of the interferogram and can be absorbed into an appropriate choice of the $y=0$ coordinate.

The original incident polarimetric spectrum is denoted $S_{j}^{o}(k)$ and represents the incident light that is being spread over the column of pixels in the FTIR. The four Stokes parameters $S_{j}$ are each formed by quadratic combinations equation 8 like $E_{x} E_{x}^{*}, B_{x} B_{x}^{*}$ and $E_{x} B_{x}^{*}$. From this feature, we can deduce the Stokes vector impinging on a pixel at position $y$ is given by

$$
S_{j}^{y}(k)=\frac{S_{j}^{o}(k)}{N} 2 \cos ^{2}\left(y k \sin \frac{\theta}{2}\right)
$$

where $k$ is the wave vector of the light under consideration.

Equation 9 shows this incident signal $S_{j}^{o}(k)$ is spread across the $N$ pixels, each pixel with 4 readouts. We will assume that each pixel responds to the light incident on its center; this means we will not average over the pixel size to get the more realistic response. Equation 9 is even in $y$; therefore $S_{j}^{-y}(k)=S_{j}^{y}(k)$, and the light hitting the pixels at $y_{o}$ and $-y_{o}$ should be the same. This observation allows us to only sample positions at $y \geq 0$ without loss of information. $N$ pixels will be able to recover $N$ evenly spaced spectral lines of information. We place pixels at $N$ discrete points between 0 and $L$ at $y_{\alpha}=d x / 2+d x(\alpha-1)$ where $\alpha$ is an index that runs from 1 through the number of pixels $N$ and $d x=L / N$.

As explained in section 1, each pixel can potentially have a very different polarization response matrix. The polarization response matrix $M_{i j}^{\alpha}(k)$ varies on the magnitude of the incident wave vector $k=2 \pi / \lambda$, and the pixel under consideration $\alpha$.

To find the response at any QWISPP on the FPA, we combine equation 9 with the polarization response matrix at each pixel's position $M_{i j}^{\alpha}(k)$ and integrate over $k$. The four responses of pixel $\alpha$ will be given by

$$
R_{i}^{\alpha}=\int d k \sum_{j=1}^{4} M_{i j}^{\alpha}(k) \frac{S_{j}^{o}(k)}{N}\left(1+\cos \left(y_{\alpha} 2 k \sin \frac{\theta}{2}\right)\right) .
$$

Generically, this spatial pattern of responses is called the interferogram.

In the next two subsections, we will discuss two techniques to recover the incident polarization spectrum $S_{j}^{o}(k)$ given four readouts at $N$ pixels $R_{i}^{\alpha}$ which describe the interferogram. The first case uses identical QWISPP pixels across the FPA with the algorithm for recovering $S_{j}^{o}\left(k_{n}\right)$ based on a traditional Fourier transform; and the second case uses randomly varying QWISPP pixels across the FPA with the algorithm for recovering $S_{j}^{o}\left(k_{n}\right)$ based on linear algebra. 


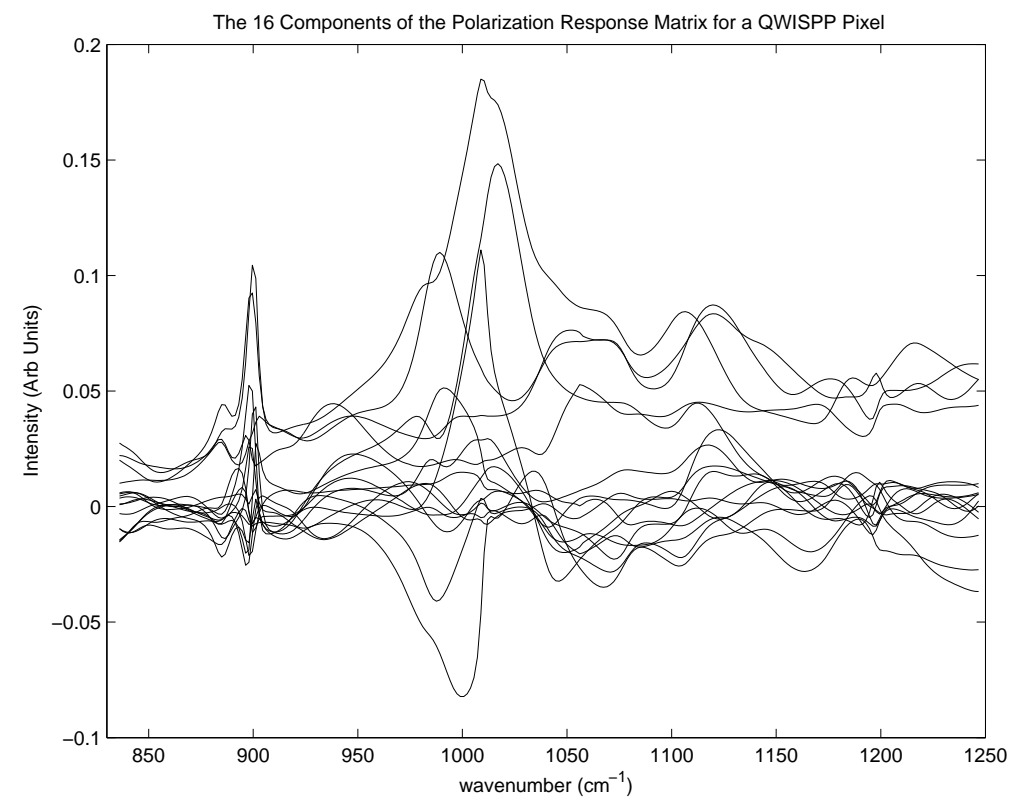

Figure 4. The polarization response matrix for a QWISPP structure varies rapidly with the wave number of the incident light.

\subsection{Recovering spectral-polarization for identical QWISPP structure across the FPA}

A slightly modified discrete Fourier transform can recover the spectral polarization in an imagining FTIR spectrometer with an FPA where every QWISPP is identical. Because we are considering identical pixels, we drop the $\alpha$ index on the polarization response matrix $M$ for this subsection.

Just like a traditional FTIR, the modes are set by the angle difference between the two beams $\theta$ and the size of the screen $L$. These $N$ modes are given by

$$
\lambda_{n}=\frac{4 L \sin (\theta / 2)}{n} \text { or } k_{n}=\frac{n \pi}{2 L \sin (\theta / 2)} .
$$

The interferogram, which is even about $y=0$, will be projected on an FPA extending from $y=0$ to $y=L$.

An actual device will receive a continuum of wavelengths of incident light. The actual response of a QWISPP will be the integral of the polarization response matrix multiplied by the incident polarization. The polarization response matrix for a QWISPP structure varies rapidly with the wave number of the incident light as shown in figure 4. The variation in $M_{i j}(k)$ with wave number is much faster than they typical variation of $S_{j}(k)$ with wave number. To accurately model the QWISPP response, the model needs to numerically integrate with a sufficient sample spacing $\Delta k$ to capture the rapidly changing polarization response matrix. This requires a data point every $\delta \lambda \approx 0.05 \mu \mathrm{m}$, or equivalently $\Delta k \approx 10 \pi \mathrm{cm}^{-1}$. One adjusts the sample spacing $\Delta k=\pi /(2 L \sin (\theta / 2))$ by changing the angle separation between beams $\theta$ or by adjusting the size $L$ of the FPA on which the interferogram is formed.

For a discrete set of modes, the integral in equation 10 becomes a sum

$$
R_{i}^{\alpha}=\sum_{n=1}^{N} \sum_{j=1}^{4} \Delta k M_{i j}\left(k_{n}\right) \frac{S_{j}^{o}\left(k_{n}\right)}{N}\left(1+\cos \left(y_{\alpha} 2 k_{n} \sin \frac{\theta}{2}\right)\right) .
$$

Using the $4 N$ readouts, we can recover at most 4 polarizations at $N$ spectral lines. We use equation 12 with $N$ spectral lines the starting point for discovering the algorithm to recover the spectral polarization. 
Orthogonality of the modes and the inverse of the polarization response matrix provide the solution

$$
S_{j}^{o}\left(k_{n}\right)=\frac{2}{\Delta k} \sum_{i=1}^{4} \sum_{\alpha=1}^{N} M_{j i}^{-1}\left(k_{n}\right) R_{i}^{\alpha} \cos \left(y_{\alpha} n \pi / L\right) .
$$

The uncertainty in the recovered Stokes vector (assuming all readouts have the same uncertainty) is given by

$$
\begin{aligned}
\delta S_{j}^{o}\left(k_{n}\right) & =\delta R \frac{2}{\Delta k} \sqrt{\sum_{i=1}^{4} \sum_{\alpha=1}^{N}\left(M_{j i}^{-1}\left(k_{n}\right)\right)^{2} \cos ^{2}\left(y_{\alpha} n \pi / L\right)} \\
& =\delta R \Lambda_{j}\left(k_{n}\right) \frac{\sqrt{2 N}}{\Delta k} .
\end{aligned}
$$

where $\Lambda_{j}\left(k_{n}\right)$ is the device's inherent polarization uncertainty at a wave number $k_{n}$ as defined in equation 3 .

An FTIR spectrometer designed with identical QWISPP pixels across the interferogram as calculated from equation 12 will suffer the same regions of uncertainty as in a grating spectrometer. The uncertainty, given in equation 12, at a particular wave number is proportional to the device's uncertainty (equation 3 ) at that same wave number. Therefore, the FTIR with identical QWISPP pixels cannot have lower uncertainty in determining the spectral polarization that the QWISPP device would have in the case of a grating spectrometer.

\subsection{Recovering spectral-polarization for varying QWISPP structure across the FPA}

There is one way to decrease the uncertainty in the recovered Stokes parameters. The key is to use a pushbroom FTIR configuration and fabricating an FPA where the QWISPP pixels vary randomly across the focal plane as described in the next subsection. QWISPP pixels with different structures will have different bands where they perform well. In a push-broom FTIR the incident light is jointly detected across a column of pixels. Therefore, by using QWISPP pixels with randomly varying structures and push-broom FTIR, we achieve a lower uncertainty in the spectral polarization.

To model this phenomena, we use the same starting point as the previous subsection (equations 11 and 12) except we return the superscript $\alpha$ on the polarization response matrix $M_{i j}^{\alpha}\left(k_{n}\right)$ to enumerate which pixel is being considered

$$
R_{i}^{\alpha}=\sum_{n=1}^{N} \sum_{j=1}^{4} \Delta k M_{j i}^{\alpha}\left(k_{n}\right)\left(1+\cos \left(y_{\alpha} 2 k_{n} \sin \frac{\theta}{2}\right)\right) \frac{S_{j}^{o}\left(k_{n}\right)}{N} .
$$

Again, $R_{i}^{\alpha}$ is again the $i^{\text {th }}$ readout at position $y_{\alpha}$. A discrete Fourier transform will not work for this case. The readouts are formed from modes multiplied by a different polarization response matrix at every pixel $\alpha$; therefore, the modes are no longer orthogonal. We can still recover the spectral-polarization using simple linear algebra at the price of some extra computer time.

Because we are using a matrix inverse and not orthogonality, one may be tempted to choose different modes than those described in equation 11, e.g modes that are not evenly spaced. Although the matrix can be inverted for different choices of modes, the error grows by many orders of magnitude for most other choices. Even with this new algorithm, remaining with the modes described in equation 11 minimizes the uncertainty in the recovered spectral polarization.

To make the simulation more realistic, we calculated the pixel response using fine sampling of the spectrum with $\Delta k^{\prime}=\Delta k / 3$ with $3 N$ spectral lines. Although we calculate the response by summing the contribution from $3 N$ spectral lines, we use equation 16 with only $N$ spectral lines as the starting point for discovering the algorithm to recover the input spectral polarization. 


\section{FTIR Simulation with Varying QWISPP Pixels Across the FPA}
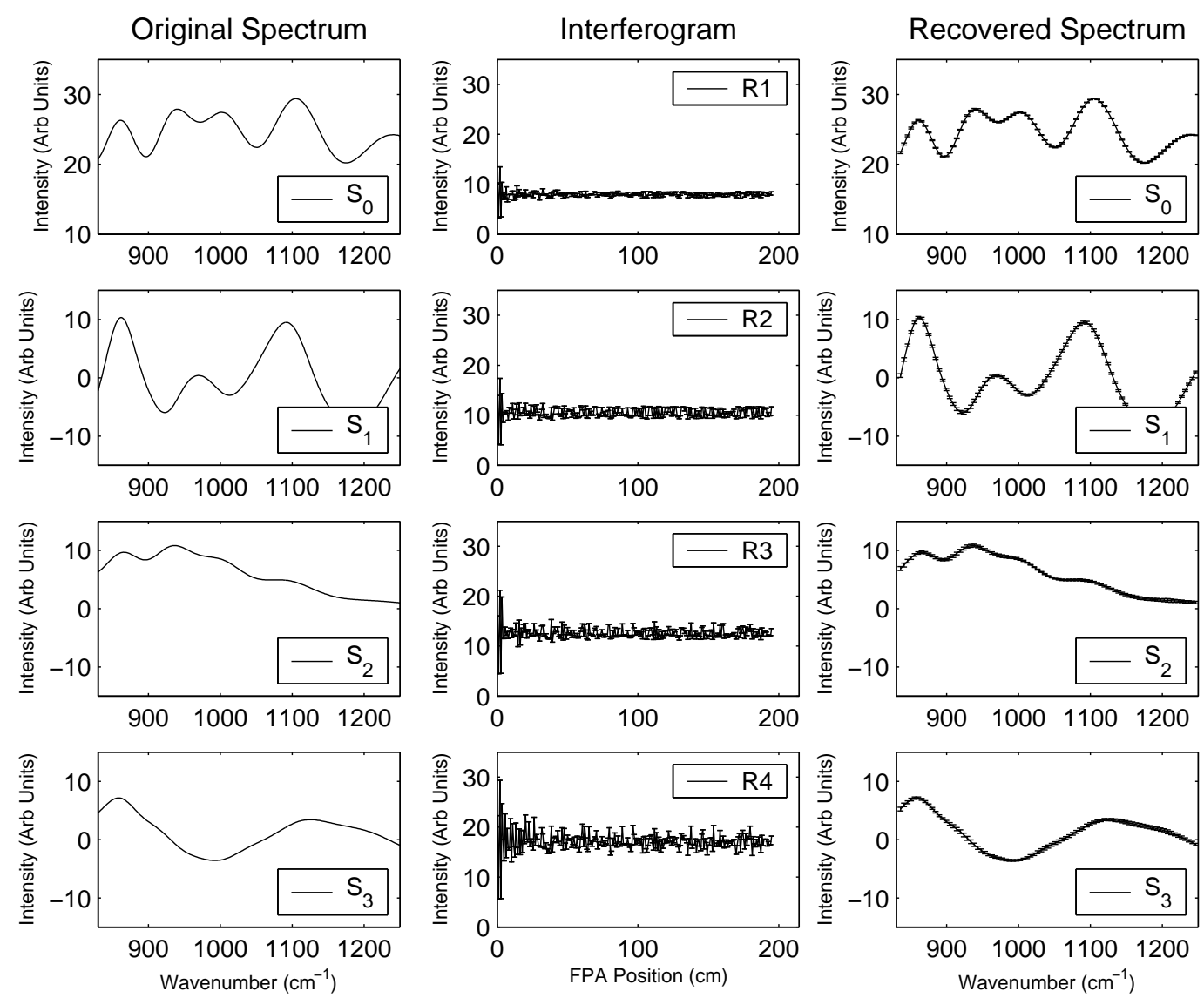

Figure 5. We modeled an FTIR with varying QWISPP pixels across the FPA. In the left column of plots, we show a hypothetical polarization spectrum. In the center column, we show the four readouts for a column of pixels in the FTIR. In the right column, we show the recovered polarization spectrum with the associated uncertainty. 
With a sufficient sampling of modes $k_{n}$, we solve this for $S_{j}^{o}\left(k_{n}\right)$ by creating a $4 N \times 4 N$ matrix $T$

$$
T_{(\alpha, i),(n, j)}=\frac{\Delta k}{N} M_{i j}^{\alpha}\left(k_{n}\right)\left(1+\cos \left(y_{\alpha} n \pi / L\right)\right)
$$

where $(\alpha, i)$ and $(n, j)$ enumerate the $4 N \times 4 N$ components. Using this matrix, we can rewrite equation 16 as

$$
R_{i}^{\alpha}=\sum_{j=1}^{4} \sum_{n=1}^{N} T_{(\alpha, i),(n, j)} S_{j}^{o}\left(k_{n}\right) .
$$

The original Stokes parameters can be recovered by inverting the matrix $T$

$$
S_{j}^{o}\left(k_{n}\right)=\sum_{i=1}^{4} \sum_{\alpha=1}^{N} T_{(n, j),(\alpha, i)}^{-1} R_{i}^{\alpha} .
$$

A typical device will not have a response at every spectral line $k_{n}$. For small $n$, the mode $k_{n}$ given by equation 11 will have a wavelength much longer than the cutoff wavelength of most detector materials. This will cause the matrix $T$ to be rectangular; in which case the pseudoinverse $\widetilde{T}^{-1}=\left(T^{T} T\right)^{-1} T^{T}$ can be used in place of a typical matrix inverse to recover the spectral polarization. Again, using the error propagation formula, the uncertainty in the recovered Stokes parameters is given by

$$
\delta S_{j}^{o}\left(k_{n}\right)=\delta R \sqrt{\sum_{i=1}^{4} \sum_{\alpha=1}^{N}\left(T_{(n, j),(\alpha, i)}^{-1}\right)^{2}} .
$$

In figure 5, we modeled an FTIR using a different QWISPP structure at each pixel of the FPA. The variation was introduced by changing the period of the top reflective grating following a normal distribution centered at 3.13 microns and with a standard deviation of 0.2 microns. In figure 5 , the left column is the incident polarization spectrum for the atmospheric window between $830-1250 \mathrm{~cm}^{-1}$ (equivalent to $8-12 \mu \mathrm{m})$. The center column shows the interferogram for each of the four readouts calculated using equation 18.

We model the readout response shown in this figure using $3 N$ spectral lines. If one does not choose a sufficiently dense set of modes $k_{n}$, recovering the spectrum becomes impossible because the actual response of a pixel, which is the integral over $k$ of the incident Stokes vector multiplied by the pixel's polarization response matrix, will deviate considerably from the modeled response from a finite sampling of modes. Our choice of $\Delta k$ places 85 spectral lines in the plotted region. Our choice of 85 spectral lines, which corresponds to $N=256$, is a dense enough sampling because our model was able to recover the incident spectrum even with the actual response formed by $3 N$ spectral lines.

Notice $R_{4}$, which is nearest the incident light, has a generally stronger response than $R_{1}$ which is near the reflective top grating of the QWISPP. In the right column of figures, the polarization spectrum is recovered using equation 19. The error bars shown on these plots are calculated from equation 20 using equation 4 for $\delta R$.

\section{DISCUSSION AND CONCLUSIONS}

The FTIR configuration shows a considerable improvement in the uncertainty of the recovered spectrum when the FPA is composed of a random sampling of QWISPP structures. To compare the improvement in uncertainty, we plot the uncertainty in the normalized Stokes parameters in figure 1.

The average uncertainty in recovering a Stokes vector improves by about $100 \times$ by using a random assortment of QWISPP pixels. For the cases we modeled, an FPA of identical QWISPPs in a grating spectrometer has an average uncertainty of 0.2416 in a normalized Stokes parameter. This uncertainty is 
shown in left side of figure 1. For the FPA with randomly varying QWISPP structures the average uncertainty drops to 0.0022 .

In addition to lower average uncertainty, we also have about a $15 \times$ improvement in the uniformity of this uncertainty across the spectral range under consideration. The standard deviation of the uncertainty is 0.1075 for the normalized Stokes parameters in the grating spectrometer pictured in left side of figure 1 . The standard deviation drops to 0.0072 in the case of randomly varying QWISPP pixels used with an FTIR.

Several benefits follow from this result. The first benefit is relaxing the manufacturing specifications for the device. The University of New Mexico, Center for High Technology Materials has a contract with the Air Force Office of Scientific Research to develop a technique to manufacture the device. Because of this result, the imperfections in manufacturing the QWISPP structure will manifest as performance improvements when used in an FTIR imager configuration.

A variation of the described QWISPP FTIR presents another benefit. An FPA with only two readouts per QWISPP pixel, but double the number of pixels, also enables full spectro-polarimetric detection. Even QWIPs with simple linear gratings at random orientations and quadruple the number of pixels will be able to detect all polarization except for $S_{3}$. In other words, other structures can be modeled and spectralpolarization recovered with the algorithm described in subsection 3.4. These devices can be built today using QWIPs with linear gratings in combination with a push-broom FTIR. These design variations sacrifice the size and weight benefits of the QWISPP in favor of designs that are easy to manufacture while retaining the benefit of near-perfect coregistration.

In this paper, we have discussed the implementation of spectral-polarimetric detection using pixels with polarization sensitivity in an FTIR configuration. We have shown that variation in the QWISPP structure can actually improve the uncertainty in recovering the polarimetric spectrum. Although the QWISPP is still a challenge to fabricate with today's technology, we have observed that the algorithms presented here can be used to easily make FTIR spectral-polarimetric detectors with current QWIP technology.

\section{ACKNOWLEDGMENTS}

We'd like to thank Dr. Michael Duggin for suggesting the idea of this paper, and we'd like to thank Dr. Carl Maes, Dr. Geoff Anderson for helpful discussions. We'd also like to thank Laura Serna and Todd Monson for reviewing the manuscript. The QWISPP idea and fabrication efforts have benefited enormously from the continued support of the Air Force Office of Scientific Research; Dr. Sanjay Krishna's research group at the University of New Mexico, Center for High Technology Materials; Dr. Z. Liau's group at MIT Lincoln Laboratory; Dr. Dave Cardimona's group at the Air Force Research Labs, Space Vehicles Directorate; and our own institution, the US Air Force Academy, Department of Physics.

\section{REFERENCES}

1. F. Sadjadi and C. Chun, "Machine recognition of objects using IR polarimetry," SPIE 2756, pp. 53-59, 1996.

2. J.Holloway, N. Witherspoon, N. Miller, K. Davis, H. Suiter, and R. Hilton, "Navy/marine corps innovative science and technology developments for future enhanced mine detection capabilities," SPIE 4038, pp. 1280-91, 2000.

3. S. H. Sposato, M. P. Fetrow, K. P. Bishop, and T. R. Caudill, "Two long-wave infrared spectral polarimeters for use in understanding polarization phenomenology," Optical Engineering 41, pp. 1055-1064, May 2002.

4. M. P. Fetrow, D. Wellems, S. H. Sposato, K. P. Bishop, T. R. Caudill, M. L. Davis, and E. R. Simrell, "Results of a new polarization simulation," in Proc. SPIE Vol. 4481, p. 149-162, Polarization Analysis, Measurement and Remote Sensing IV, Dennis H. Goldstein; David B. Chenault; Walter G. Egan; Michael J. Duggin; Eds., pp. 149-162, Jan. 2002. 
5. M. Serna, "Quantum-well detector concept for hyperspectral coregistered full Stokes vector detection," in Photonics for Space Environments VIII. Edited by Taylor, Edward W. Proceedings of the SPIE, Volume 4823, pp. 113-123 (2002)., pp. 113-123, Sept. 2002.

6. D. A. Cardimona, D. H. Huang, D. T. Le, H. S. Gingrich, and M. A. Serna, "Advanced IR detector devices and concepts for remote sensing," in Infrared Spaceborne Remote Sensing XI. Edited by Strojnik, Marija. Proceedings of the SPIE, Volume 5152, pp. 316-326 (2003)., pp. 316-326, Dec. 2003.

7. M. Serna, "Model and fabrication of a proof-of-concept polarimeter-in-a-pixel," Master's Thesis, University of New Mexico, Electrical Engineering Department (ArXiv Physics e-prints physics/0406080), July 2003.

8. L. J. Otten, B. A. Jones, A. Edwards, J. Lane, T. R. Caudill, V. Osweiler, S. Yarbrough, J. Arnold, R. Quarles, and E. T. Kouba, "Space-based hyperspectral imagery of the moon and Earth limb," in Proc. SPIE Vol. 4540, p. 88-99, Sensors, Systems, and Next-Generation Satellites V, Hiroyuki Fujisada; Joan B. Lurie; Konradin Weber; Eds., pp. 88-99, Dec. 2001.

9. S. Yarbrough, T. R. Caudill, E. T. Kouba, V. Osweiler, J. Arnold, R. Quarles, J. Russell, L. J. Otten, B. A. Jones, A. Edwards, J. Lane, A. D. Meigs, R. B. Lockwood, and P. S. Armstrong, "MightySat II.1 hyperspectral imager: summary of on-orbit performance," in Proc. SPIE Vol. 4480, p. 186-197, Imaging Spectrometry VII, Michael R. Descour; Sylvia S. Shen; Eds., pp. 186-197, Jan. 2002.

10. F. Iannarilli, S. Jones, H. Scott, and P. Kebabian, "Polarimetric-spectral intensity modulation (PSIM): Enabling simultaneous hyperspectral and polarimetric imaging," SPIE Conference on Infrared Technology Applications XXV 3698, p. 474, April 1999.

11. D. Beekman and J. V. Anda, "Polarization sensitive QWIP thermal imager," Infrared Physics and Technology 42, pp. 323-328, 2001.

12. J. Peterson, G. Jensen, and J. Kristl, "Imaging polarimetry capabilities and measurement uncertainties in remote sensing applicaitons," SPIE 4133, pp. 221-232, 2000.

13. J. V. Delden, "Ortho-babinet polarization-interrogating filter: an interferometric approach to polarization measurement," Optics Letters 28, pp. 1173-1176, July 2003.

14. M. Serna, "Single-pixel polarimeter: Dielectric-gratings model and fabrication progress," Infrared Physics and Technology 44, pp. 457-464, 2003.

15. J. Y. Andersson and L. Lundqvist, "Near-unity quantum efficiency of AlGaAs/GaAs quantum-well infrared detectors using a waveguide with a doubly periodic grating coupler," Appl. Phys. Lett. 59(7), p. 857, 1991.

16. S. J. Andersson and L. Lundqvist, "Grating-coupled quantum-well infrared detectors: Theory and performance," J. Appl. Phys. 71(7), p. 3600, 1992.

17. M. Jhabvala, K. Choi, A. C. Goldberg, A. T. La, and S. D. Gunapala, "Development of a $1 \mathrm{~K} \times 1 \mathrm{~K}$ GaAs QWIP far IR imaging array," in Focal Plane Arrays for Space Telescopes. Edited by Grycewicz, Thomas J.; McCreight, Craig R. Proceedings of the SPIE, Volume 5167, pp. 175-185 (2004)., pp. 175-185, Jan. 2004.

18. J. Pan and C. Fonstad, "Theory, fabrication and characterization of quantum-well infrared photodetectors," Material Science and Engineering 28, pp. 65-147, 2000. 